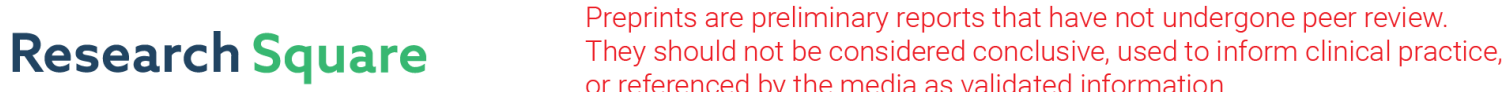 or referenced by the media as validated information. \\ Boundary Control of Stochastic Korteweg-de Vries- Burgers Equations
}

\section{Shuang Liang}

Harbin Institute of Technology Weihai

Kai-Ning Wu ( $\nabla$ wkn@hit.edu.cn )

Harbin Institute of Technology Weihai https://orcid.org/0000-0003-4122-9832

\section{Research Article}

Keywords: Stochastic Korteweg-de Vries-Burgers equations, boundary control, robust stabilization, $\mathrm{H}$ infinity control

Posted Date: January 25th, 2022

DOI: https://doi.org/10.21203/rs.3.rs-1274504/v1

License: (1) This work is licensed under a Creative Commons Attribution 4.0 International License.

Read Full License

Version of Record: A version of this preprint was published at Nonlinear Dynamics on March 30th, 2022.

See the published version at https://doi.org/10.1007/s11071-022-07398-3. 


\title{
Boundary control of stochastic Korteweg-de Vries-Burgers equations
}

\author{
Shuang Liang · Kai-Ning Wu
}

Received: date / Accepted: date

\begin{abstract}
The boundary control problem is considered for stochastic Korteweg-de Vries-Burgers equations. First, a boundary controller is proposed, and a criterion is obtained for mean square exponential stability by using Lyapunov functional method and inequality techniques. Then, when there exist uncertainties in the system parameters, the robust mean square exponential stability is considered, and a sufficient criterion is obtained. Furthermore, if there are also additive noises in the considered system, the H-infinity performance is investigated and a sufficient condition is obtained to ensure the mean square H-infinity performance. Numerical examples illustrate the validity of the theoretical results.
\end{abstract}

Keywords Stochastic Korteweg-de Vries-Burgers equations · boundary control · robust stabilization · H-infinity control

\section{Introduction}

Korteweg-de Vries (KdV) equations are a class of partial differential equations of shallow water wave with unidirectional motion, and they are also a typical representative of nonlinear dispersive equations. Burgers equations can simulate the propagation and reflection of shock waves, and they are also a typical representative of nonlinear dissipative equations. The study of $\mathrm{KdV}$ equations or Burgers equations has been an active research topic because of its potential applications $[3$, $4,6,22,28,40]$.

Corresponding author: Kai-Ning Wu. Email:wkn@hit.edu.cn.

S. Liang, K. N. Wu

Department of Mathematics, Harbin Institute of Technology,

Weihai, 264209, China

Tel.: +86-631-5687035; Fax: +86-631-5687572

E-mail: liangshuang20180@163.com (S. Liang)
In many actual physical problems, the case of pure dispersion and pure dissipation is really rare. When adding a diffusion term, the KdV equation becomes Korteweg-de Vries-Burgers (KdVB) equation. KdVB equations are widely applied in the fields of engineering, fluid mechanics, biological mathematics, and so on. For example, in fluid mechanics, the corresponding KdVB equation is

$$
y_{t}(x, t)-\varepsilon y_{x x}(x, t)+\delta y_{x x x}(x, t)+y y_{x}(x, t)=0,
$$

where $y(x, t)$ represents the height from the horizontal plane to the free surface of water wave, $t$ represents time, $x$ represents the distance of water wave moving along the direction of propagation, $\varepsilon$ is the dissipation coefficient and $\delta$ is the dispersion coefficient.

For partial differential equations, the control problem has been a research hotspot $[10,21,23,30,37,41]$. Generally, the control strategies for partial differential systems can be divided into two categories, distributed control and boundary control. Distributed control means that the actuators need to be placed at each point of the spatial domain, which is not easy to realize in engineering [18]. Boundary control only needs to apply the actuators on the boundary of the spatial region, which has the characteristics of low cost and easy to realize. In recent years, boundary control has been studied by many scholars $[1,5,8,9,12,13]$. For the boundary control of deterministic systems, a classic method is the backstepping method. Many researchers have made a lot of excellent results for deterministic KdVB equations which have taken on an upsurge [2,32-34]. Global boundary stabilization of KdVB equations was studied in [25]. Smaoui et al. proposed a boundary control strategy for the generalized KdVB equations on the interval $[0,1]$ in $[31]$. For the boundary control of stochastic systems, it is difficult to use the backstepping method. 
There exists an essential difficulty in dealing with the Itô's formula. Then, it is worth considering to use Lyapunov functional method for the boundary control of stochastic systems.

In real applications, there are uncertainties of system parameters in modeling, which will naturally affect the behavior analysis of the system. Since parameter uncertainties may destroy the stability, the robust stability for partial differential equations is an interesting topic in control system analysis $[15,26]$. Sakthivel studied the problem of robust global stabilization by a nonlinear boundary feedback control for KdVB equations on the domain $[0,1]$ in [29]. Moreover, there may also be external noises, which enter the system in the form of non-Gaussian additive noises, and they will also affect the behavior of the system. H-infinity control is a feasible strategy to reduce the effect of external disturbances on system states (or systems output), which has been widely concerned [11,20,24,38,39]. In [19], Kang et al. considered the observer-based H-infinity control problem for a stochastic KdVB equation under point or averaged measurements.

Random noises are ubiquitous. KdVB equations may be disturbed by random environmental factors, so it is important to study stochastic KdVB (SKdVB) equations. Stochastic systems driven by Brownian motions, have received extensive attention in population growth, electronic circuits, financial investment and so on. The stability of this kind of systems has been an important subject $[7,16,17]$. However, the results on the stability or stabilization of SKdVB equations are still few.

There will be many difficulties and challenges when studying the boundary control of SKdVB equations. It is difficult to deal with the non-linear terms in SKdVB equations. The boundary control strategy only applies a controller on the boundary of the spatial region and does not directly affect the state equations of the system. Using the general systems analysis methods, it is difficult to analyze the behavior of the system. In addition, due to the addition of stochastic items, it brings extra difficulties to the performance analysis of the system. Moreover, when the single-point controller in [25] is used to study the stability of SKdVB equations. A criterion of stability is obtained. It is suitable for small noises and small disturbances. This limits the application of the theoretical results. Meanwhile, when the integral controller in [36] is used to study the stability of SKdVB equations. It can't handle the non-linear items in the system. Therefore, for SKdVB equations, we should present a novel controller design scheme.

Basing on the above discussions, we aim to investigate the boundary control for SKdVB equations. A boundary controller is designed first. By Lyapunov func- tional method and inequality techniques, a sufficient criterion is established to ensure the mean square exponential stability. Then, when there exist uncertainties in the system parameters, the robust boundary stabilization is considered. In addition, when the system is disturbed by external additive noises, a criterion is presented to ensure the mean square $\mathrm{H}$-infinity performance under our designed boundary controller. The major contributions are listed as follows.

1. A boundary controller is designed and a sufficient condition is presented to achieve mean square exponential stability of SKdVB equations under the designed boundary controller.

2. A criterion is obtained to achieve robust stabilization of the uncertain SKdVB equations.

3. A criterion is obtained to achieve mean square finite horizon H-infinity performance of SKdVB equations with additive noises.

Notation: Throughout this paper, $W^{l, 2}\left([0, L] ; \mathbb{R}^{n}\right)$ stands for a Sobolev space of absolutely continuous functions $\sigma(x):[0, L] \rightarrow \mathbb{R}^{n}$ with square integrable derivatives $\frac{d^{l} \sigma(x)}{d x^{l}}$ up to order $l . \mathbb{R}^{n}$ is the $n$-dimensional Euclidean space. $\mathcal{L}^{2}(0, l)$ stands for the Hilbert space of square integrable scalar functions over $(0, l)$ with the corresponding norm $\|z\|_{\mathcal{L}^{2}(0, l)}^{2}=\int_{0}^{l}|z(x)|^{2} \mathrm{~d} x$. Let $(\Omega, \mathcal{F}, \mathbb{P})$ be a complete probability space adapted to the filtration $\left\{\mathcal{F}_{t}\right\}_{t \geq 0}$ satisfying the usual conditions. $\mathbb{E}$ denotes the mathematical expectation with respect the probability measure $\mathbb{P}$.

\section{Preliminaries and model formulation}

We consider the following SKdVB equation

$$
\left\{\begin{aligned}
& \mathrm{d} y(x, t)= {\left[\varepsilon y_{x x}(x, t)-\delta y_{x x x}(x, t)-y y_{x}(x, t)\right] \mathrm{d} t } \\
&+C y(x, t) \mathrm{d} W(t), t>0, x \in(0,1), \\
& y(0, t)= y_{x}(1, t)=0, y_{x x}(1, t)=u(t), \\
& y(x, 0)=\phi(x),
\end{aligned}\right.
$$

where $\varepsilon, \delta$ and $C$ are positive constants, $y(x, t)$ is the state, $x$ and $t$ are the space variable and time variable, $\phi(x)$ is the initial state of the system, and $u(t)$ is the boundary control input, $W(t)$ is the one-dimensional standard Brownian motion, which satisfies

$$
\mathbb{E}(\mathrm{d} W(t))=0, \mathbb{E}(\mathrm{d} W(t))^{2}=\mathrm{d} t
$$

For the further analysis, the following definition and lemmas are needed. 
Definition 1 ([19]) System (1) is said to be mean square exponentially stable if there exist positive numbers $M>0$ and $\eta>0$ such that

$$
\mathbb{E}\|y(\cdot, t)\|^{2} \leq M\|\phi(\cdot)\|^{2} e^{-\eta t}, \quad t \geq 0
$$

for all $\phi(x) \in L^{2}(0,1)$, where $\|y(\cdot, t)\|^{2}=\int_{0}^{1} y^{2}(x, t) \mathrm{d} x$.

Lemma 1 (Poincaré's Inequality [14]) Let $z \in W^{1,2}$ $\left([0, L] ; \mathbb{R}^{n}\right)$ be a vector function with $z(0)=0$ or $z(L)=$ 0 . Then, for a matrix $R>0$, we have the following integral inequality

$\int_{0}^{L} z^{\mathrm{T}}(s) R z(s) \mathrm{d} s \leq \frac{4 L^{2}}{\pi^{2}} \int_{0}^{L}\left(\frac{\mathrm{d} z(s)}{\mathrm{d} s}\right)^{\mathrm{T}} R\left(\frac{\mathrm{d} z(s)}{\mathrm{d} s}\right) \mathrm{d} s$.

Lemma 2 (Itô's formula [27]) Let $\mathcal{X}_{t}$ be an Itô progress with the stochastic differential $\mathrm{d} \mathcal{X}_{t}=\Gamma_{t} \mathrm{~d} t+Q_{t} \mathrm{~d} B_{t}$. Suppose that $\nu(x) \in C^{2}(\mathbb{R})$ and $\nu\left(\mathcal{X}_{t}\right) \in \mathcal{L}^{2}$. Then $Y_{t}=\nu\left(\mathcal{X}_{t}\right)$ is also an Itô progress and satisfies

$$
\mathrm{d} Y_{t}=\frac{\partial \nu}{\partial x}\left(\mathcal{X}_{t}\right) \mathrm{d} \mathcal{X}_{t}+\frac{1}{2} \frac{\partial^{2} \nu}{\partial x^{2}}\left(\mathcal{X}_{t}\right)\left(\mathrm{d} \mathcal{X}_{t}\right)^{2}
$$

Moreover,

$$
\mathrm{d} Y_{t}=\left(\frac{\partial \nu}{\partial x}\left(\mathcal{X}_{t}\right) \Gamma_{t}+\frac{1}{2} \frac{\partial^{2} \nu}{\partial x^{2}} Q_{t}^{2}\right) d t+\frac{\partial \nu}{\partial x}\left(\mathcal{X}_{t}\right) Q_{t} \mathrm{~d} B_{t}
$$

Lemma 3 (Gronwall Inequality [27]) Let $u(\cdot)$ be a Borel measurable bounded nonnegative function on $\left[t_{0}, T\right]$, and let $v(\cdot)$ be a non-negative integrable function on $\left[t_{0}, T\right]$. If for any scalars $c \geq 0$ and $t \in\left[t_{0}, T\right]$, we have

$$
u(t) \leq c+\int_{t_{0}}^{t} v(s) u(s) \mathrm{d} s
$$

then

$$
u(t) \leq c \exp \left(\int_{t_{0}}^{t} v(s) d s\right), \text { for all } t \in\left[t_{0}, T\right]
$$

\section{Main results}

In this section, first, we design a boundary controller and obtain a sufficient condition to ensure the mean square exponential stabilization for SKdVB equations. Then, the robust stabilization is studied for the uncertain SKdVB equations. Finally, H-infnity control is investigated for the disturbed SKdVB equations.

\subsection{Mean square exponential stabilization}

In this subsection, a boundary controller is designed for system (1). Based on the designed controller, a sufficient condition is obtained to ensure the mean square exponential stabilization.

We take the following boundary controller for system (1)

$u(t)= \begin{cases}K_{1} y^{2}(1, t)-\frac{K_{2}}{y(1, t)} \int_{0}^{1} y^{2}(x, t) \mathrm{d} x, & y(1, t) \neq 0, \\ 0, & y(1, t)=0\end{cases}$

where $K_{1}$ and $K_{2}$ are the boundary control gain.

We present the following theorem to guarantee that system (1) achieves the mean square exponential stability under the boundary controller (2).

Theorem 1 If constants $K_{1}$ and $K_{2}$ satisfy the following inequalities

$K_{1}<-\frac{1}{3 \delta}, \quad K_{2}<-\frac{C^{2}}{2 \delta}$,

then system (1) under the boundary controller (2) achieves mean square exponential stability.

Proof Consider the following functional

$V(t)=V(y(\cdot, t))=\int_{0}^{1} y^{2}(x, t) \mathrm{d} x$.

Then, along system (1), we have

$$
\begin{aligned}
\mathrm{d} V(t)= & \left\{\int_{0}^{1}\left[2 y\left(\varepsilon y_{x x}-\delta y_{x x x}-y y_{x}\right)+C^{2} y^{2}\right] \mathrm{d} x\right\} \mathrm{d} t \\
& +\left(2 \int_{0}^{1} C y^{2} \mathrm{~d} x\right) \mathrm{d} W(t) .
\end{aligned}
$$

Using integration by parts and the boundary conditions of system (1), we obtain that

$$
\begin{aligned}
\mathrm{d} V(t)= & \left\{\left.2 \varepsilon y y_{x}\right|_{0} ^{1}-2 \varepsilon \int_{0}^{1} y_{x}^{2} \mathrm{~d} x-\left.2 \delta y y_{x x}\right|_{0} ^{1}\right. \\
& \left.+2 \delta \int_{0}^{1} y_{x} y_{x x} \mathrm{~d} x-\left.\frac{2}{3} y^{3}\right|_{0} ^{1}+\int_{0}^{1} C^{2} y^{2} \mathrm{~d} x\right\} \mathrm{d} t \\
& +\left(2 \int_{0}^{1} C y^{2} \mathrm{~d} x\right) \mathrm{d} W(t) \\
= & \left\{-2 \varepsilon \int_{0}^{1} y_{x}^{2} \mathrm{~d} x-\left.2 \delta y y_{x x}\right|_{0} ^{1}+\left.\delta y_{x}^{2}\right|_{0} ^{1}-\left.\frac{2}{3} y^{3}\right|_{0} ^{1}\right. \\
& \left.+\int_{0}^{1} C^{2} y^{2} \mathrm{~d} x\right\} \mathrm{d} t+\left(2 \int_{0}^{1} C y^{2} \mathrm{~d} x\right) \mathrm{d} W(t) .
\end{aligned}
$$


Noting $y(0, t)=0$, by virtue of Lemma 1 , we obtain

$-2 \varepsilon \int_{0}^{1} y_{x}^{2} \mathrm{~d} x \leq-\frac{\varepsilon \pi^{2}}{2} \int_{0}^{1} y^{2} \mathrm{~d} x$.

Substituting (6) into (5), we have

$$
\begin{aligned}
\mathrm{d} V(t)= & \left\{-2 \varepsilon \int_{0}^{1} y_{x}^{2} \mathrm{~d} x-2 \delta y(1, t) u(t)-\delta y_{x}^{2}(0, t)\right. \\
& \left.-\frac{2}{3} y^{3}(1, t)+\int_{0}^{1} C^{2} y^{2} \mathrm{~d} x\right\} \mathrm{d} t \\
& +\left(2 \int_{0}^{1} C y^{2} \mathrm{~d} x\right) \mathrm{d} W(t) \\
\leq & \left\{-2 \varepsilon \int_{0}^{1} y_{x}^{2} \mathrm{~d} x-2 \delta y(1, t)\left[K_{1} y^{2}(1, t)\right.\right. \\
& \left.-\frac{K_{2}}{y(1, t)} \int_{0}^{1} y^{2} \mathrm{~d} x\right]-\delta y_{x}^{2}(0, t)-\frac{2}{3} y^{3}(1, t) \\
& \left.+\int_{0}^{1} C^{2} y^{2} \mathrm{~d} x\right\} \mathrm{d} t+\left(2 \int_{0}^{1} C y^{2} \mathrm{~d} x\right) \mathrm{d} W(t) \\
\leq & \left\{\int _ { 0 } ^ { 1 } \left[-\frac{\varepsilon \pi^{2}}{2} y^{2}+\left(\delta K_{1}+\frac{1}{3}\right)\left(y^{2}(1, t)\right.\right.\right. \\
& \left.\left.\left.+y^{4}(1, t)\right)+\left(2 \delta K_{2}+C^{2}\right) y^{2}\right] \mathrm{~d} x\right\} \mathrm{d} t \\
& +\left(2 \int_{0}^{1} C y^{2} \mathrm{~d} x\right) \mathrm{d} W(t) .
\end{aligned}
$$

Using conditon (3), we have

$\mathrm{d} V(t) \leq-\lambda \int_{0}^{1} y^{2} \mathrm{~d} x \mathrm{~d} t+\left(2 \int_{0}^{1} C y^{2} \mathrm{~d} x\right) \mathrm{d} W(t)$,

where $\lambda=\frac{\varepsilon \pi^{2}}{2}$.

In light of Lemma 3 , we obtain

$$
\begin{aligned}
& \mathbb{E}\left[e^{\lambda t} V(t)\right]-\mathbb{E}\left[e^{\lambda_{0}} V(0)\right]=\mathbb{E} \int_{0}^{t} \mathrm{~d}\left[e^{\lambda s} V(s)\right] \\
& =\mathbb{E} \int_{0}^{t} \lambda e^{\lambda s} V(s) \mathrm{d} s+\mathbb{E} \int_{0}^{t} e^{\lambda s} \mathrm{~d} V(s) .
\end{aligned}
$$

Substituting (8) into (9), we have

$$
\begin{aligned}
\mathbb{E}\left[e^{\lambda t} V(t)\right] & \leq \\
& V(0)+\mathbb{E} \int_{0}^{t}\left[\lambda e^{\lambda s} V(s)-\lambda e^{\lambda s} \int_{0}^{1} y^{2} \mathrm{~d} x\right] \mathrm{d} s \\
& +\mathbb{E} \int_{0}^{t}\left[2 e^{\lambda s} \int_{0}^{1} C y^{2}(x, s) \mathrm{d} x\right] \mathrm{d} W(s) \\
& =V(0) .
\end{aligned}
$$

Thus, we obtain

$\mathbb{E} V(t) \leq V(0) e^{-\lambda t}$,

that is,

$\mathbb{E}\|y(\cdot, t)\|^{2} \leq\|\phi(\cdot)\|^{2} e^{-\lambda t}$,

which completes the proof.

Remark 1 For SKdVB equations, we present a novel controller design scheme (2) instead of using the singlepoint controller or the integral controller alone which was adopted in $[25,36]$. The reasons are as follows. When the single-point controller $u(t)=k_{1} y^{3}(1, t)+k_{2} y(1, t)$ in [25] is used to study the stability of system (1), a stability criterion is obtained. We can get the following inequality must be held: $C^{2}-\frac{\varepsilon \pi^{2}}{2}<0$, and this restriction implies that this criterion is workable only for small random interference $C$. This limits the application of the theoretical results, and many systems may not meet this criterion, so this design is inappropriate. Meanwhile, the integral controller $u(t)=K \int_{0}^{1} y(x, t) \mathrm{d} x$ in [36] is can't handle the non-linear items in system (1). Therefore, the pure integral boundary controller is not workable here.

Remark 2 It should be noted that we design the controller (2) with integral form in order to suppress the item $C^{2}$. The existence of $y^{2}(1, t)$ in controller design is to deal with the third order term in system (1) on the stability.

Remark 3 In system (1), according to criterion (3), we see that the larger the dispersion coefficient $\delta$, the more difficult it is to find the satisfied $K_{1}$. In the real applications, the dispersion coefficient is equivalent to the speed of water wave, that is, the faster the amplitude of water wave, the more difficult to make it stable. At the same time, the larger $C$ means the large random disturbances, and the more difficult it is to find the satisfied $K_{2}$, the more difficult it is to stabilize the system.

\subsection{Robust stabilization for SKdVB equations}

In this subsection, a sufficient condition is derived for robust stabilization of SKdVB equations under our designed boundary controller (2).

When the system (1) have uncertainties in the parameters $\varepsilon$ and $C$, and we assume that they belong to the interval of

$$
\begin{aligned}
& \varepsilon \in\left[\varepsilon_{0}-\frac{1}{\eta_{1}}, \varepsilon_{0}+\frac{1}{\eta_{1}}\right], \\
& C \in\left[C_{0}-\frac{1}{\eta_{2}}, C_{0}+\frac{1}{\eta_{2}}\right],
\end{aligned}
$$


where $\varepsilon_{0}>0, C_{0}>0, \eta_{1}>0$ and $\eta_{2}>0$ are given constants, $\varepsilon_{0}$ and $C_{0}$ represent the nominal value (see, for instance, [29]), $\frac{1}{\eta_{1}}$ and $\frac{1}{\eta_{2}}$ represent the maximum magnitude of the allowable uncertainty, respectively.

We rewrite system (1) as

$$
\begin{gathered}
\mathrm{d} y(x, t)=\left[\left(\varepsilon_{0}+\frac{\lambda}{\eta_{1}}\right) y_{x x}(x, t)-\delta y_{x x x}(x, t)-y y_{x}(x, t)\right] \\
\mathrm{d} t+\left(C_{0}+\frac{\lambda}{\eta_{2}}\right) y(x, t) \mathrm{d} W(t),
\end{gathered}
$$

where $\lambda \in[-1,1]$ represents the uncertainty.

In order to derive a sufficient condition for mean square exponential stability of the uncertain system (14), we introduce new signals $z, \omega$ and let $z=y_{x x}$, $\omega=\lambda z$. The system (14) is written as

$$
\begin{gathered}
\mathrm{d} y(x, t)=\left[\varepsilon_{0} y_{x x}(x, t)+\frac{\omega}{\eta_{1}}-\delta y_{x x x}(x, t)-y y_{x}(x, t)\right] \\
\mathrm{d} t+\left(C_{0}+\frac{\lambda}{\eta_{2}}\right) y(x, t) \mathrm{d} W(t),
\end{gathered}
$$

where $\omega$ represents the uncertainty.

For the subsequent discussion, the following lemma about parameter uncertainties is given.

Lemma 4 Let $\alpha=\left|\frac{y}{2 \eta_{1} y_{x x}}\right|$, then for $z=y_{x x}, \omega=\lambda z$, we have the following inequality

$\alpha z^{2}-\alpha \omega^{2} \geq 0$

which is guaranteed by $\lambda \in[-1,1]$.

Proof The proof is motivated by the one in [29]. From $z=y_{x x}$, we have

$\alpha z^{2}=\alpha y_{x x}^{2}=\left|\frac{y y_{x x}}{2 \eta_{1}}\right|$.

From $\omega=\lambda z$, we have

$\alpha \omega^{2}=\alpha \lambda^{2} y_{x x}^{2}=\left|\frac{\lambda^{2} y y_{x x}}{2 \eta_{1}}\right|$.

Substituting (17) and (18) into (16),

$\alpha z^{2}-\alpha \omega^{2}=\left|\frac{\left(1-\lambda^{2}\right) y y_{x x}}{2 \eta_{1}}\right| \geq 0$,

which is guaranteed by $\lambda \in[-1,1]$.

This proof is complete.

Theorem 2 Suppose that constans $K_{1}$ and $K_{2}$ satisfy the following inequalities

$K_{1}<-\frac{2}{3 \delta}, \quad K_{2}<-\frac{1}{\delta}\left(C_{0}+\frac{1}{\eta_{2}}\right)^{2}$,

then uncertain system (14) with the boundary controller (2) achieves mean square exponential stability.
Proof Consider the following functional

$V(t)=V(y(\cdot, t))=\frac{1}{2} \int_{0}^{1} y^{2}(x, t) \mathrm{d} x$.

Then, along system (14) and using inequality (16), we have

$$
\begin{aligned}
\mathrm{d} V(t)= & \left\{\int _ { 0 } ^ { 1 } \left[y\left(\varepsilon_{0} y_{x x}+\frac{\lambda}{\eta_{1}} y_{x x}-\delta y_{x x x}-y y_{x}\right)\right.\right. \\
& \left.\left.+\left(C_{0}+\frac{\lambda}{\eta_{2}}\right)^{2} y^{2}\right] \mathrm{~d} x\right\} \mathrm{d} t \\
& +\left(2 \int_{0}^{1}\left(C_{0}+\frac{\lambda}{\eta_{2}}\right) y^{2} \mathrm{~d} x\right) \mathrm{d} W(t) \\
\leq & \left\{\int _ { 0 } ^ { 1 } \left[y\left(\varepsilon_{0} y_{x x}+\frac{\lambda}{\eta_{1}} y_{x x}-\delta y_{x x x}-y y_{x}\right)\right.\right. \\
& \left.\left.+\left(C_{0}+\frac{\lambda}{\eta_{2}}\right)^{2} y^{2}\right] \mathrm{~d} x+\alpha z^{2}-\alpha \omega^{2}\right\} \mathrm{d} t \\
& +\left(2 \int_{0}^{1}\left(C_{0}+\frac{\lambda}{\eta_{2}}\right) y^{2} \mathrm{~d} x\right) \mathrm{d} W(t) .
\end{aligned}
$$

Using integration by parts, applying the completion of squares to (22) and the boundary conditions of system (14), we have

$$
\begin{aligned}
\mathrm{d} V(t) \leq & \left\{\varepsilon_{0} \int_{0}^{1} y y_{x x} \mathrm{~d} x+\int_{0}^{1}\left(\frac{1}{4 \eta_{1}^{2} \alpha} y^{2}+\alpha y_{x x}^{2}\right) \mathrm{d} x\right. \\
& -\left.\delta y y_{x x}\right|_{0} ^{1}+\left.\frac{1}{2} \delta y_{x}^{2}\right|_{0} ^{1}-\left.\frac{1}{3} y^{3}\right|_{0} ^{1}+\int_{0}^{1}\left(C_{0}+\frac{\lambda}{\eta_{2}}\right)^{2} \\
& \left.y^{2} \mathrm{~d} x\right\} \mathrm{d} t+\left(2 \int_{0}^{1}\left(C_{0}+\frac{\lambda}{\eta_{2}}\right) y^{2} \mathrm{~d} x\right) \mathrm{d} W(t) \\
\leq & \left\{\left(\frac{\varepsilon_{0} \eta_{1}+1}{\eta_{1}}\right) \int_{0}^{1} y y_{x x} \mathrm{~d} x-\delta y(1, t) u(t)\right. \\
& -\frac{1}{2} \delta y_{x}^{2}(0, t)-\frac{1}{3} y^{3}(1, t)+\int_{0}^{1}\left(C_{0}+\frac{\lambda}{\eta_{2}}\right)^{2} \\
& \left.y^{2} \mathrm{~d} x\right\} \mathrm{d} t+\left(2 \int_{0}^{1}\left(C_{0}+\frac{\lambda}{\eta_{2}}\right) y^{2} \mathrm{~d} x\right) \mathrm{d} W(t) \\
= & \left\{-\left(\frac{\varepsilon_{0} \eta_{1}+1}{\eta_{1}}\right) \int_{0}^{1} y_{x}^{2} \mathrm{~d} x-\delta y(1, t) u(t)\right. \\
& -\frac{1}{2} \delta y_{x}^{2}(0, t)-\frac{1}{3} y^{3}(1, t)+\int_{0}^{1}\left(C_{0}+\frac{\lambda}{\eta_{2}}\right)^{2} \\
& \left.y^{2} \mathrm{~d} x\right\} \mathrm{d} t+\left(2 \int_{0}^{1}\left(C_{0}+\frac{\lambda}{\eta_{2}}\right) y^{2} \mathrm{~d} x\right) \mathrm{d} W(t) .
\end{aligned}
$$

Noting $y(0, t)=0$, by virtue of Lemma 1 , we obtain

$$
-\left(\frac{\varepsilon_{0} \eta_{1}+1}{\eta_{1}}\right) \int_{0}^{1} y_{x}^{2} \mathrm{~d} x \leq-\frac{\left(\varepsilon_{0} \eta_{1}+1\right) \pi^{2}}{4 \eta_{1}} \int_{0}^{1} y^{2} \mathrm{~d} x .
$$


Substituting (24) into (23), we have

$$
\begin{aligned}
\mathrm{d} V(t) \leq & -\left(\frac{\varepsilon_{0} \eta_{1}+1}{\eta_{1}}\right) \int_{0}^{1} y_{x}^{2} \mathrm{~d} x-\delta y(1, t)\left[K_{1} y^{2}(1, t)\right. \\
& \left.-\frac{K_{2}}{y(1, t)} \int_{0}^{1} y^{2} \mathrm{~d} x\right]-\frac{1}{3} y^{3}(1, t)+\int_{0}^{1}\left(C_{0}+\right. \\
& \left.\left.\frac{\lambda}{\eta_{2}}\right)^{2} y^{2} \mathrm{~d} x\right\} \mathrm{d} t+\left(2 \int_{0}^{1}\left(C_{0}+\frac{\lambda}{\eta_{2}}\right) y^{2} \mathrm{~d} x\right) \mathrm{d} W(t) \\
\leq & \left\{\int _ { 0 } ^ { 1 } \left[-\frac{\left(\varepsilon_{0} \eta_{1}+1\right) \pi^{2}}{4 \eta_{1}} y^{2}+\left(\frac{1}{2} \delta K_{1}+\frac{1}{3}\right)\right.\right. \\
& \left(y^{2}(1, t)+y^{4}(1, t)\right)+\left(\left(C_{0}+\frac{\lambda}{\eta_{2}}\right)^{2}+\delta K_{2}\right) \\
& \left.\left.y^{2}\right] \mathrm{~d} x\right\} \mathrm{d} t+\left(2 \int_{0}^{1}\left(C_{0}+\frac{\lambda}{\eta_{2}}\right) y^{2} \mathrm{~d} x\right) \mathrm{d} W(t) .
\end{aligned}
$$

Using conditon (20), we have

$$
\begin{aligned}
\mathrm{d} V(t) \leq-\tilde{\lambda} \int_{0}^{1} y^{2} \mathrm{~d} x \mathrm{~d} t+\left(2 \int_{0}^{1}\left(C_{0}+\frac{\lambda}{\eta_{2}}\right)\right. \\
\left.y^{2} \mathrm{~d} x\right) \mathrm{d} W(t),
\end{aligned}
$$

where $\tilde{\lambda}=\frac{\left(\varepsilon_{0} \eta_{1}+1\right) \pi^{2}}{4 \eta_{1}}$.

Hence, the uncertain terms were eliminated. Following a similar line to that of Theorem 1, and the results can be carried out.

Remark 4 The introduction of the scaling $\alpha$ enables us to eliminate the uncertainty $\lambda$ in the time-derivative of the Lyapunov function, which is motivated by the one in $[29]$.

\subsection{H-infinity control for SKdVB equations}

When non-Gaussian additive noises enter SKdVB equations, properties of the system may be degraded or even destroyed. Under this situation, we need to study the abilities of the system to resist the external disturbances, and that is the H-infinity control problem.

When system (1) is subject to exogenous disturbances, it turns into

$$
\begin{aligned}
\mathrm{d} y(x, t)= & {\left[\varepsilon y_{x x}(x, t)-\delta y_{x x x}(x, t)-y y_{x}(x, t)+\nu(x, t)\right] } \\
& \mathrm{d} t+C y(x, t) \mathrm{d} W(t), t>0, x \in(0,1),
\end{aligned}
$$

where $\nu(x, t)$ is the external disturbance, and we assume that

$$
\int_{0}^{t_{f}} \int_{0}^{1} \nu^{2}(x, t) \mathrm{d} x \mathrm{~d} t<\infty
$$

for a positive time constant $t_{f}$.

Now, we present the definition of mean square $\mathrm{H}$ infinity performance for system (27).

Definition 2 ([35]) System (27) ahiceves mean square $\mathrm{H}$-infinity performance over the finite horizon $\left[0, t_{f}\right]$ if for a given positive time constant $0<t_{f}<\infty$ and a disturbance attenuation level $\gamma>0$, when $y(x, 0)=0$, the following inequality holds

$\mathbb{E}\left(\int_{0}^{t_{f}} \int_{0}^{1} y^{2} \mathrm{~d} x \mathrm{~d} t\right) \leq \gamma^{2} \mathbb{E}\left(\int_{0}^{t_{f}} \int_{0}^{1} \nu^{2} \mathrm{~d} x \mathrm{~d} t\right)$.

The following theorem gives the result of the $\mathrm{H}$ infinity control for system (27).

Theorem 3 Assume that constants $K_{1}$ and $K_{2}$ satisfying

$K_{1}<-\frac{1}{3 \delta}, \quad K_{2}<-\frac{1}{2 \delta}\left(1+\gamma^{-2}+C^{2}\right)$,

then system (27) with the boundary control (2) has mean square H-infinity performance over the finite horizon $\left[0, t_{f}\right]$.

Proof Taking the Lyapunov functional as the one defined in (4), and computing $\mathrm{d} V$ along system (27) yields

$$
\begin{aligned}
\mathrm{d} V(t)= & \left\{\int_{0}^{1}\left[2 y\left(\varepsilon y_{x x}-\delta y_{x x x}-y y_{x}+\nu\right)\right] \mathrm{d} x\right\} \mathrm{d} t \\
& +\left(2 \int_{0}^{1} C y^{2} \mathrm{~d} x\right) \mathrm{d} W(t) .
\end{aligned}
$$

For the given constants $\gamma$ and $t_{f}$, using the fact that $V(y(\cdot, 0))=0$ when $y(x, 0)=0$, we have

$$
\begin{aligned}
\mathbb{E} \int_{0}^{t_{f}} & \int_{0}^{1}\left(y^{2}-\gamma^{2} \nu^{2}\right) \mathrm{d} x \mathrm{~d} t \\
= & \mathbb{E} \int_{0}^{t_{f}} \int_{0}^{1}\left(y^{2}-\gamma^{2} \nu^{2}\right) \mathrm{d} x \mathrm{~d} t+\mathbb{E} \int_{0}^{t_{f}} \mathrm{~d} V \\
& +\mathbb{E} V(y(\cdot, 0))-\mathbb{E} V\left(y\left(\cdot, t_{f}\right)\right) \\
\leq & \mathbb{E} \int_{0}^{t_{f}} \int_{0}^{1}\left(y^{2}-\gamma^{2} \nu^{2}\right) \mathrm{d} x \mathrm{~d} t+\mathbb{E} \int_{0}^{t_{f}} \int_{0}^{1}[2 y \\
& \left.\left(\varepsilon y_{x x}-\delta y_{x x x}-y y_{x}+\nu\right)+C^{2} y^{2}\right] \mathrm{d} x \mathrm{~d} t \\
= & \mathbb{E} \int_{0}^{t_{f}} \int_{0}^{1}\left[\left[-\gamma^{2}\left(\nu-\gamma^{-2} y\right)^{2}\right]+y^{2}+\gamma^{-2} y^{2}\right. \\
& \left.+2 y\left(\varepsilon y_{x x}-\delta y_{x x x}-y y_{x}+\nu\right)+C^{2} y^{2}\right] \mathrm{d} x \mathrm{~d} t \\
\leq & \mathbb{E} \int_{0}^{t_{f}} \int_{0}^{1}\left[y^{2}+\gamma^{-2} y^{2}+2 y\left(\varepsilon y_{x x}-\delta y_{x x x}\right.\right. \\
& \left.\left.-y y_{x}+\nu\right)+C^{2} y^{2}\right] \mathrm{d} x \mathrm{~d} t .
\end{aligned}
$$


It follows from (7) that

$$
\begin{aligned}
\mathbb{E} \int_{0}^{t_{f}} & \int_{0}^{1}\left(y^{2}-\gamma^{2} \nu^{2}\right) \mathrm{d} x \mathrm{~d} t \\
\leq & \mathbb{E} \int_{0}^{t_{f}} \int_{0}^{1}\left[y^{2}+\gamma^{-2} y^{2}-\frac{\varepsilon \pi^{2}}{2} y^{2}+\left(\delta K_{1}+\frac{1}{3}\right)\right. \\
& \left.\left(y^{2}(1, t)+y^{4}(1, t)\right)+\left(2 \delta K_{2}+C^{2}\right) y^{2}\right] \mathrm{d} x \mathrm{~d} t \\
= & \mathbb{E} \int_{0}^{t_{f}} \int_{0}^{1}\left[-\frac{\varepsilon \pi^{2}}{2} y^{2}+\left(\delta K_{1}+\frac{1}{3}\right)\left(y^{2}(1, t)\right.\right. \\
& \left.\left.+y^{4}(1, t)\right)+\left(1+\gamma^{-2}+2 \delta K_{2}+C^{2}\right) y^{2}\right] \mathrm{d} x \mathrm{~d} t .
\end{aligned}
$$

By using the techniques as in the proof of Theorem 1 , condition (29) is equivalent

$\mathbb{E} \int_{0}^{t_{f}} \int_{0}^{1}\left(y^{2}-\gamma^{2} \nu^{2}\right) \mathrm{d} x \mathrm{~d} t \leq 0$,

which completes our proof.

\section{Numerical example}

In this section, we give three numerical examples to verify the effectiveness of our results.

Example 1 We consider a SKdVB equation

$$
\begin{aligned}
\mathrm{d} y(x, t)= & {\left[6 y_{x x}(x, t)-0.05 y_{x x x}(x, t)-y y_{x}(x, t)\right] \mathrm{d} t } \\
& +0.1 y(x, t) \mathrm{d} W(t), t>0, x \in(0,1) .
\end{aligned}
$$

The initial value is taken as

$y(x, 0)=\frac{1}{6} x^{3}-\frac{1}{2} x^{2}+\frac{1}{2} x$.

The boundary conditions are

$y(0, t)=y_{x}(1, t)=0, y_{x x}(1, t)=u(t)$.

We adopt the following boundary controller

$u(t)= \begin{cases}K_{1} y^{2}(1, t)-\frac{K_{2}}{y(1, t)} \int_{0}^{1} y^{2}(x, t) \mathrm{d} x, & y(1, t) \neq 0 \\ 0, & y(1, t)=0\end{cases}$

Let $K_{1}=-7, K_{2}=-3$, we can verify that condition (3) holds. By virtue of Theorem 1, we know that system (33) achieves mean square exponential stability. Under the boundary controller (36), the mean-square system state $\mathbb{E} y^{2}(x, t)$, as shown in Fig. 1 is mean-square exponential stable. Moreover, Fig. 2 shows the mean-square state norm of system (33).

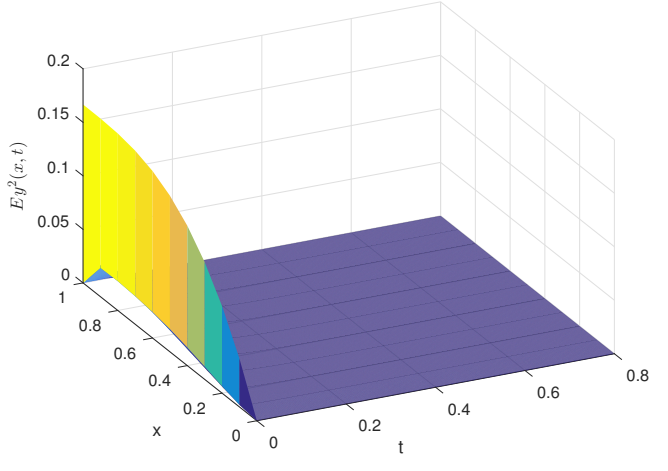

Fig. 1 State curve $\mathbb{E} y^{2}(x, t)$ of system (33)

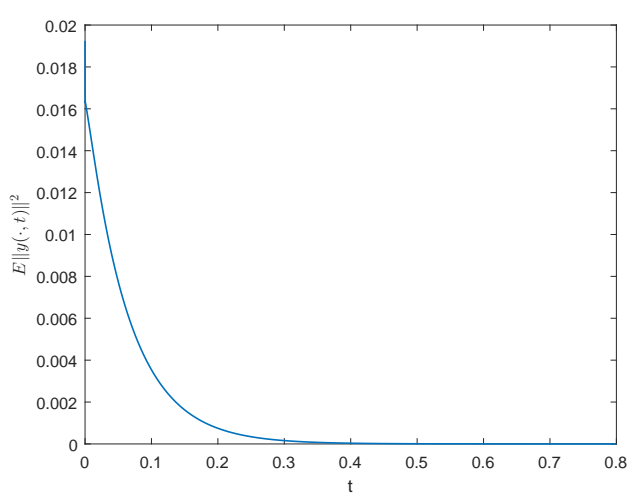

Fig. 2 State norm $\mathbb{E}\|y(\cdot, t)\|^{2}$ of system (33)

Example 2 Consider the following uncertain SKdVB equation

$$
\begin{aligned}
\mathrm{d} y(x, t)=[( & \left.+\frac{\lambda}{2}\right) y_{x x}(x, t)-0.04 y_{x x x}(x, t) \\
& \left.-y y_{x}(x, t)\right] \mathrm{d} t+\left(0.4+\frac{\lambda}{5}\right) y(x, t) \mathrm{d} W(t), \\
& t>0, x \in(0,1) .
\end{aligned}
$$

The initial value is taken as

$$
y(x, 0)=\frac{1}{9} x^{3}-\frac{1}{2} x^{2}+\frac{1}{3} x .
$$

The boundary conditions are

$y(0, t)=y_{x}(1, t)=0, y_{x x}(1, t)=u(t)$.

We adopt the following boundary controller

$u(t)= \begin{cases}K_{1} y^{2}(1, t)-\frac{K_{2}}{y(1, t)} \int_{0}^{1} y^{2}(x, t) \mathrm{d} x, & y(1, t) \neq 0 \\ 0, & y(1, t)=0\end{cases}$

Let $K_{1}=-17, K_{2}=-6$, we can verify that condition (20) holds. By virtue of Theorem 2, we know that 
uncertain system (37) achieves mean square exponential stability.

For the numerical simulation, we choose the unknown parameter $\lambda=\sin (\pi x t)$. We observe that system (37) achieves robust mean square exponential stability. Fig. 3 shows the state in mean square sense of system (37). Moreover, Fig.4 shows $\mathbb{E}\|y(\cdot, t)\|^{2}$ under the boundary controller (40).

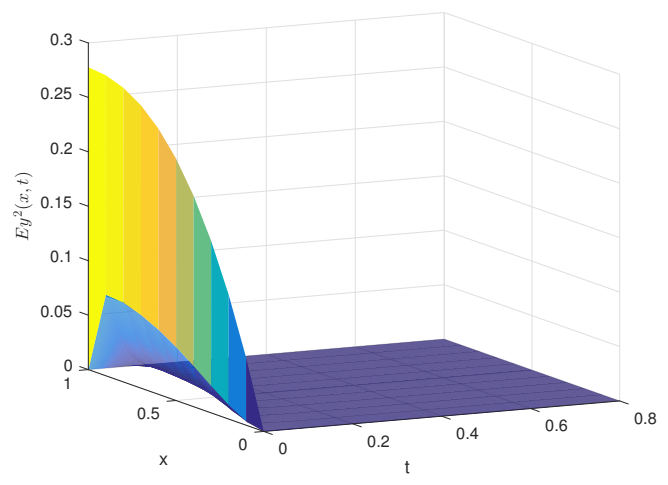

Fig. 3 State curve $\mathbb{E} y^{2}(x, t)$ of uncertain system (37)

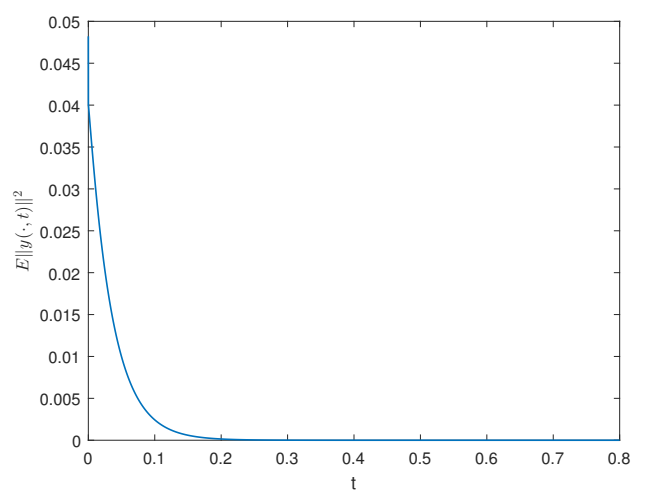

Fig. 4 State norm $\mathbb{E}\|y(\cdot, t)\|^{2}$ of uncertain system (37)

Example 3 Consider a SKdVB equation with external disturbances as follows

$$
\begin{aligned}
\mathrm{d} y(x, t)=[ & 2 y_{x x}(x, t)-0.03 y_{x x x}(x, t)-y y_{x}(x, t) \\
& +\nu(x, t)] \mathrm{d} t+0.2 y(x, t) \mathrm{d} W(t), \\
& t>0, x \in(0,1) .
\end{aligned}
$$

The boundary conditions are

$y(0, t)=y_{x}(1, t)=0, y_{x x}(1, t)=u(t)$.
We adopt the following boundary controller

$u(t)= \begin{cases}K_{1} y^{2}(1, t)-\frac{K_{2}}{y(1, t)} \int_{0}^{1} y^{2}(x, t) \mathrm{d} x, & y(1, t) \neq 0, \\ 0, & y(1, t)=0 .\end{cases}$

The disturbance function $\nu(x, t)$ is choosen as $\nu(x, t)=1.75 \cos (0.3 \pi t)+\sin (3 x)$.

Set the disturbed attenuation level $\gamma=0.9$, and $t_{f}=5$. Under the initial value $y(x, 0)=0$, the following ratio is calculated when taking 100 sample paths,

$$
\frac{\mathbb{E} \int_{0}^{5} \int_{0}^{1} y^{2} \mathrm{~d} x \mathrm{~d} t}{\mathbb{E} \int_{0}^{5} \int_{0}^{1} \nu^{2} \mathrm{~d} x \mathrm{~d} t}=0.6167^{2}<0.9^{2}=\gamma^{2}
$$

Let $K_{1}=-12, K_{2}=-38$, we can verify that condition (29) holds. By virtue of Theorem 3, we know that disturbed system (41) achieves mean square H-infinity performance over the finite horizon $[0,5]$.

\section{Conclusions}

In this paper, boundary control for SKdVB equations has been addressed. First, we design a boundary controller, and a sufficient condition is obtained for mean square exponential stability by applying Lyapunov functional method and inequality techniques. Then, the boundary controller is applied to achieve robust mean square exponential stability for uncertain SKdVB equations, and the corresponding sufficient condition is obtained. In addition, if there are also additive noises in the considered system, the H-infinity performance is investigated and a sufficient condition is obtained to ensure the H-infinity performance in the mean square sense. Finally numerical simulations show the validity of the obtained results.

Acknowledgements This work was supported by Natural Science Foundations of Shandong Provence under Grant ZR2021MF007.

\section{Conflict of Interest}

The authors declare that there is no conflict of interest.

\section{Data availability}

The data that support the findings of this study are available from the corresponding author upon reasonable request. 


\section{References}

1. Baccoli, A., Pisano, A., Orlov, Y.: Boundary control of coupled reaction-diffusion processes with constant parameters. Automatica 54, 80-90 (2015)

2. Balogh, A., Krstic, M.: Boundary control of the Korteweg-de Vries-Burgers equation: further results on stabilization and well-posedness, with numerical demonstration. IEEE Transactions on Automatic Control 45(9), 1739-1745 (2000)

3. Baudouin, L., Crepeau, E., Valein, J.: Two approaches for the stabilization of nonlinear KdV equation with boundary time-delay feedback. IEEE Transactions on Automatic Control 64(4), 1403-1414 (2019)

4. Burns, J.A., Kang, S.: A control problem for Burgers' equation with bounded input/output. Nonlinear Dynamics 2, 235-262 (1991)

5. Cai, X.S., Krstic, M.: Nonlinear control under wave actuator dynamics with time- and state-dependent moving boundary. International Journal of Robust \& Nonlinear Control 25(2), 222-251 (2015)

6. Cerpa, E., Coron, J.M.: Rapid stabilization for a Korteweg-de Vries equation from the left Dirichlet boundary condition. IEEE Transactions on Automatic Control 58(7), 1688-1695 (2013)

7. Chanthorn, P., Rajchakit, G., Thipcha, J., Emharuethai, C., Sriraman, R., Lim, C.P., Ramachandran, R.: Robust stability of complex-valued stochastic neural networks with time-varying delays and parameter uncertainties. IET Control Theory \& Applications 8(5), 742 (2020)

8. Cheng, Y., Wu, Y.H., Guo, B.Z.: Absolute boundary stabilization for an axially moving Kirchhoff beam. Automatica 129, 109,667 (2021)

9. Ding, K., Zhu, Q.X., Liu, L.J.: Extended dissipativity stabilization and synchronization of uncertain stochastic reaction-diffusion neural networks via intermittent non-fragile control. Journal of the Franklin InstituteEngineering and Applied Mathematics 356(18), 11,69011,715 (2019)

10. Fridman, E., Orlov, Y.: Exponential stability of linear distributed parameter systems with time-varying delays. Automatica 45(1), 194-201 (2009)

11. Gao, Q., Feng, G., Xi, Z.Y., Wang, Y., Qiu, J.B.: Robust $H_{\infty}$ control of T-S fuzzy time-delay systems via a new sliding-mode control scheme. IEEE Transactions on Fuzzy Systems 22(2), 459-465 (2014)

12. Han, X.X., Wu, K.N., Ding, X.H., Yang, B.Q.: Boundary control of stochastic reaction-diffusion systems with Markovian switching. International Journal of Robust and Nonlinear Control 30(10), 4129-4148 (2020)

13. Hashimoto, T., Krstic, M.: Stabilization of reaction diffusion equations with state delay using boundary control input. IEEE Transactions on Automatic Control 61(12), 4041-4047 (2016)

14. Heinonen, J.: Lectures on Analysis on Metric Spaces. Springer, New York (2001)

15. Hu, L.J., Mao, X.R., Zhang, L.G.: Robust stability and boundedness of nonlinear hybrid stochastic differential delay equations. IEEE Transactions on Automatic Control 58(9), 2319-2332 (2013)

16. $\mathrm{Hu}, \mathrm{W} ., \mathrm{Zhu}, \mathrm{Q} . \mathrm{X}$. : Moment exponential stability of stochastic nonlinear delay systems with impulse effects at random times. International Journal of Robust \& Nonlinear Control 29(12), 3809-3820 (2019)

17. Humphries, U., Rajchakit, G., Kaewmesri, P., Chanthorn, P., Sriraman, R., Samidurai, R., Lim, C.P.:
Stochastic memristive quaternion-valued neural networks with time delays: An analysis on mean square exponential input-to-state stability. Mathematics 8(5), 815 (2020)

18. Kang, W., Fridman, E.: Distributed stabilization of Korteweg-de Vries-Burgers equation in the presence of input delay. Automatica 100, 260-273 (2019)

19. Kang, W., Wang, X.N., Wu, K.N., Li, Q., Liu, Z.J.: Observer-based $H_{\infty}$ control of a stochastic Kortewegde Vries-Burgers equation. International Journal of Robust and Nonlinear Control 31(12), 5943-5961 (2021)

20. Kao, Y.G., Li, W., Wang, C.H.: Nonfragile observerbased $H_{\infty}$ sliding mode control for Ito stochastic systems with Markovian switching. International Journal of Robust \& Nonlinear Control 24(15), 2035-2047 (2014)

21. Krstic, M., Smyshlyaev, A.: Adaptive boundary control for unstable parabolic PDEs-Part 1: Lyapunov Design. IEEE Transactions on Automatic Control 53(7), 15751591 (2008)

22. Li, Z.P., Qin, Q.Q., Li, W.Z., Xu, S.Z., Qian, Y.Q., Sun, J.: Stabilization analysis and modified KdV equation of a car-following model with consideration of self-stabilizing control in historical traffic data. Nonlinear Dynamics 91(2), 1113-1125 (2018)

23. Lin, Y., Zhang, Y.: Stochastic stability of non-linear impulsive semi-Markov jump systems. IET Control Theory \& Applications 13(11), 1753-1760 (2019)

24. Liu, L.J., Chen, W.H., Lu, X.M.: Impulsive $H_{\infty}$ synchronization for reaction-diffusion neural networks with mixed delays. Neurocomputing 272, 481-494 (2018)

25. Liu, W.J., Krstic, M.: Global boundary stabilization of the Korteweg-de Vries-Burgers equation. Computational \& Applied Mathematics 21(1), 315-354 (2002)

26. Lou, X.Y., Cui, B.T.: Robust exponential stabilization of a class of delayed neural networks with reaction-diffusion terms. International Journal of Neural Systems 16(6), 435-443 (2006)

27. Mao, X.R.: Stochastic Differential Equations and Applications, 2nd edn. Horwood Publishing Limited, Chichester (2007)

28. Marx, S., Cerpa, E.: Output feedback stabilization of the Korteweg-de Vries equation. Automatica 87, 210-217 (2018)

29. Sakthivel, R.: Robust stabilization the Kortewegde Vries-Burgers equation by boundary control. Nonlinear Dynamics 58(4), 739-744 (2009)

30. Shang, Y.F., Xu, G.Q.: Stabilization of an EulerBernoulli beam with input delay in the boundary control. Systems \& Control Letters 61(11), 1069-1078 (2012)

31. Smaoui, N., Al-Jamal, R.: Boundary control of the generalized Korteweg-de Vries-Burgers equation. Nonlinear Dynamics 51(3), 439-446 (2008)

32. Smaoui, N., Al-Jamal, R.: Dynamics and control of the modified generalized Korteweg-de Vries-Burgers equation with periodic boundary conditions. Nonlinear Dynamics 103(1), 987-1009 (2021)

33. Smaoui, N., El-Kadri, A., Zribi, M.: Nonlinear boundary control of the unforced generalized Korteweg-de VriesBurgers equation. Nonlinear Dynamics 60(4), 561-574 (2010)

34. Smaoui, N., El-Kadri, A., Zribi, M.: Adaptive boundary control of the unforced generalized Korteweg-de VriesBurgers equation. Nonlinear Dynamics 69(3), 1237-1253 (2012)

35. Wang, J.W., Wu, H.N., Sun, C.Y.: Local exponential stabilization via boundary feedback controllers for a class of unstable semi-linear parabolic distributed parameter processes. Journal of the Franklin Institute-Engineering and Applied Mathematics 354(13), 5221-5244 (2017) 
36. Wu, K.N., Liu, X.Z., Peng, S., Cheng, C.L.: Boundary control of linear stochastic reaction-diffusion systems. International Journal of Robust and Nonlinear Control 29(1), 268-282 (2019)

37. Wu, K.N., Sun, H.X., Peng, S., Cheng, C.L.: Finitetime boundary stabilization of reaction-diffusion systems. International Journal of Robust and Nonlinear Control 28(5), 1641-1652 (2018)

38. Wu, S.N., Wen, S.H.: Robust $H_{\infty}$ output feedback control for attitude stabilization of a flexible spacecraft. Nonlinear Dynamics 84(1), 405-412 (2016)

39. Xu, S.Y., Chen, T.W.: Robust $H_{\infty}$ control for uncertain discrete-time stochastic bilinear systems with Markovian switching. International Journal of Robust and Nonlinear Control 15(5), 201-217 (2005)

40. Yang, X.J., Machado, J., Hristov, J.: Nonlinear dynamics for local fractional Burgers' equation arising in fractal flow. Nonlinear Dynamics 84(1), 3-7 (2016)

41. Zhang, C.L., Deng, F.Q., Peng, Y.J., Zhang, B.: Adaptive synchronization of Cohen-Grossberg neural network with mixed time-varying delays and stochastic perturbation. Applied Mathematics and Computation 269, 792$801(2015)$ 